\title{
Increased neutrophil-to-lymphocyte ratio is a novel marker for nutrition, inflammation and chemotherapy outcome in patients with locally advanced and metastatic esophageal squamous cell carcinoma
}

\author{
YU SATO $^{1,2}$, KENJI GONDA $^{2,3}$, MAIKO HARADA $^{4}$, YUKI TANISAKA $^{4}$, SHIN ARAI $^{4}$, \\ YUMI MASHIMO ${ }^{4}$, HIROTOSHI IWANO ${ }^{4}$, HIROSHI SATO ${ }^{5}$, SHOMEI RYOZAWA ${ }^{4}$, \\ TAKAO TAKAHASHI ${ }^{6}$, SHINICHI SAKURAMOTO ${ }^{5}$ and MASAHIKO SHIBATA ${ }^{7}$ \\ ${ }^{1}$ Department of Surgery, Toho University Sakura Medical Center, Sakura, Chiba 285-8741; \\ ${ }^{2}$ Department of Gastroenterological Oncology, Saitama Medical University International Medical Center, Hidaka, \\ Saitama 350-1298; ${ }^{3}$ Department of Surgery, Japan Community Healthcare Organization, Nihonmatsu Hospital, \\ Nihonmatsu, Fukushima 964-8501; Departments of ${ }^{4}$ Gastroenterology, ${ }^{5}$ Surgery and ${ }^{6}$ Palliative Medicine, \\ Saitama Medical University International Medical Center, Hidaka, Saitama 350-1298; ${ }^{7}$ Department of Advanced \\ Cancer Immunotherapy, Fukushima Medical University, Fukushima, Fukushima 960-1259, Japan
}

Received April 18, 2017; Accepted May 22, 2017

DOI: $10.3892 /$ br.2017.924

\begin{abstract}
Esophageal squamous cell carcinoma (ESCC) is one of the most common types of cancer, and its progression is strongly influenced by the presence of inflammation. Recently, there has been growing interest in the host inflammatory response, and increasing evidence has indicated that the neutrophil-to-lymphocyte ratio (NLR), a useful marker of systemic inflammation, may be an effective prognostic indicator in various types of malignant diseases. In the present study, 260 patients with ESCC were enrolled, including 110 who received chemoradiation therapy (CRT) involving irradiation and chemotherapy of 5-fluorouracil and cisplatin, and 150 received chemotherapy using 5 -fluorouracil and cisplatin (FP). The patients of each group were both divided into two groups according to their NLR: High NLR (NLR>3.0) and low NLR $(\mathrm{NLR} \leq 3.0)$. Serum levels of prealbumin and retinol binding protein, which are nutritional parameters, were both significantly inversely correlated with NLR in patients treated with CRT, and patients treated with FP. Levels of CRP, a marker of inflammation, were significantly correlated with NLR, and stimulation indices, markers of immune reactions, were inversely correlated with NLR in both of CRT patients and FP patients. In patients treated with CRT, a partial response
\end{abstract}

Correspondence to: Dr Kenji Gonda, Department of Surgery, Japan Community Healthcare Organization, Nihonmatsu Hospital, 1-553 Naritamachi, Nihonmatsu, Fukushima 964-8501, Japan E-mail: gonken@lime.plala.or.jp

Key words: neutrophil-to-lymphocyte ratio, esophageal squamous cell carcinoma, nutrition, inflammation was significantly higher in patients with a low NLR and with progressive disease compared to those with a high NLR. In patients treated with FP, a partial response was also significantly higher in patients with a low NLR and with progressive disease compared to those with a high NLR. The overall survival of patients with CRT and FP were both significantly worse in patients with a high NLR than in those with a low NLR.NLR may serve as a useful marker of the tumor response, immune suppression, malnutrition and prognosis upon CRT or FP in patients with locally advanced or metastatic ESCC.

\section{Introduction}

Cancers of the esophagus are placed among the most common malignancies worldwide, being the eighth leading cause of cancer related deaths (1). Esophageal squamous cell cancer (ESCC) is a dominant histological type of esophageal malignancies $(1,2)$. Previously, there has been growing interest in investigating the host inflammatory response to tumors, and systemic inflammatory responses reflect the stimulation of angiogenesis, DNA damage and tumor invasion caused by the overproduction of cytokines (3-5). Thus, the prognostic value of inflammation-based prognostic scores, such as the Glasgow Prognostic Score (GPS) and the platelet-to-lymphocyte ratio $(6,7)$, has been studied. In addition, increasing evidence has indicated that the neutrophil-to-lymphocyte ratio (NLR) may be an effective prognostic indicator in various types of malignant diseases (8-18).

ESCC is characterized by profound immune dysfunction thought to be caused by cytokine production originating from tumor cells and host immune competent cells (19-21). In cancer patients, lymphopenia is the surrogate of impaired cell-mediated immunity, whereas neutrophilia is a response to systemic inflammation. NLR is considered to be a marker of general 
immune responses to various stress stimuli. It correlates with the severity of clinical progress in severely ill patients in the intensive care unit and emerging evidence suggests that it may have prognostic value in patients with solid tumors (22-24).

Because $>60 \%$ of newly diagnosed ESCC patients present either locally advanced or metastatic disease, surgical resection is not applied in these patients. For these patients, a chemoradiation therapy (CRT)-based multidisciplinary treatment may be the only approach to achieve cure instead of an esophagectomy $(1,2,25)$. Surgical resection has constituted the primary treatment option in the treatment of esophageal cancer. Despite advances in surgical methodologies, long-term survival following surgery alone for advanced esophageal cancer has remained poor. The implementation of perioperative chemotherapy has improved survival rates. The most commonly applied regimen for CRT and neoadjuvant chemotherapy is the combination of cisplatin and 5-fluorouracil (FP) (25-27).

Currently, it is widely accepted that inflammatory cells, which are present in the tumor microenvironment, and the tumor immune response, which is activated by ionizing radiation, significantly affect tumor development (28-31). This tumor-generated inflammatory response may result in an increased propensity for apoptosis, angiogenesis and DNA damage by upregulating cytokines and inflammatory mediators.

Cancer-induced cachexia, a complex metabolic syndrome associated with underlying illness, is another significant problem in cancer treatment and care as it is associated with poor responses to chemotherapy, nutritional impairment and immune suppression $(32,33)$. The authors previously reported that malnutrition or hypoalbuminemia correlates well with immune suppression, systemic inflammation and suppression of cell-mediated immunity (34-38). Systemic inflammation may underlie these conditions, which are prominent in patients with advanced cancer. Thus, the relationship between the NLR and nutritional status or immune function was also assessed in the current study.

\section{Materials and methods}

Study population. The authors enrolled 260 patients with locally advanced and metastatic ESCC including 110 patients treated with CRT and 150 with FP, were treated between May 2013 and May 2016 at Saitama Medical University International Medical Center (Hidaka, China). In patients with disturbance of oral nutrition, CRT was mainly applied. Patient characteristics are listed in Table I. The study was approved by the Institutional Review Board of Saitama Medical University International Medical Center (Hidaka, China). All of the patients provided written informed consent. Patient age ranged from 41 to 79 years (median, 65.3 years) in the CRT group and 40 to 78 years (median, 63.6 years) in the FP group, and Eastern Cooperative Study Group PS was 0 or 1. Peripheral blood were obtained before any treatments, and NLR was defined as follows: Percentage of neutrophils in white blood cells (WBCs)/percentage of lymphocytes in WBCs or peripheral neutrophil count/peripheral lymphocyte count. To assess the cell-mediated response, phytohemagglutinin was used to stimulate lymphocyte proliferation, followed by a lymphocyte
Table I. Patient characteristics.

\begin{tabular}{lcc}
\hline Characteristics & CRT & FP \\
\hline Patients (n) & 110 & 150 \\
Age & $65.3(41-79)$ & $63.6(40-78)$ \\
NLR $>3$ & 57 & 61 \\
NLR $\leq 3$ & 53 & 89 \\
\hline
\end{tabular}

CRT, chemoradiation therapy; FP, 5-fluorouracil and cisplatin; NLR, neutrophil-to-lymphocyte ratio.

proliferation assay in which proliferation was measured by $3 \mathrm{H}$-thymidine incorporation; the proliferative response was expressed as the stimulation index (SI). The serum concentrations of prealbumin (PA) and retinol-binding protein (RBP), rapid turnover proteins, were measured as nutritional parameters by immunoturbidimetry and latex flocculation turbidimetry, respectively, and serum levels of C-reactive protein (CRP) were measured by latex flocculation turbidimetry as an indicator of inflammation. The patients of CRT group and FP group were divided into two groups with 3.0 of NLR. CRT patients included 57 with a high NLR and 53 with a low NLR and FP patients did 61 with a high NLR and 89 with a low NLR (Table I).

Chemotherapy regimen. For CRT treatment, all radiation treatments were delivered as either 3D-CRT or intensity-modulated radiation therapy with standard fractionation (i.e., 1.8/2.0 Gy fractions once daily for 5 days/week). Radiation was delivered by high-energy (6 or $15 \mathrm{MV}$ ) linear accelerators. Patients were treated with a total dose of 50-64 Gy administered over 25-32 fractions. Chemotherapy began on day 1 concurrent with the initial radiation treatments, and the regimens included two cycles of 5-fluorouracil and cisplatin. 5-fluorouracil $\left(700 \mathrm{mg} / \mathrm{m}^{2}\right)$ was administered intravenously (i.v.) on days 1-3, and cisplatin $\left(25 \mathrm{mg} / \mathrm{m}^{2}\right)$ was administered by i.v. infusion in $500 \mathrm{ml}$ either normal saline or dextrose on days 1-3. For FP treatment, two cycles of cisplatin and 5-fluorouracil (5-FU), which consisted of $80 \mathrm{mg} / \mathrm{m}^{2}$ cisplatin administered on days 1 and 29 and 800 $\mathrm{mg} / \mathrm{m}^{2} / 24 \mathrm{~h}$ 5-FU administered on days 1-3 and days 29-31. The responses were evaluated according to the Response Evaluation Criteria in Solid Tumors (RECIST) version 1.0 (39) following two cycles of treatment. The patients who responded to therapy received surgical resection and the same chemotherapy regimen again, and those who did not respond to treatment received docetaxel as second-line treatment.

Statistical analysis. The significance among parameters including NLR was analyzed by the $\chi^{2}$ test and Student's t-test. The strength and significance of correlations were assessed by Spearman's rank correlation coefficient. Among the patients entered in the study, 104 patients with CRT and 145 patients with FP were available for the analysis of survival. The survival curves of patients who received CRT or FP were estimated using the Kaplan-Meier method, and the log-rank test were used to compare the survival curves. $\mathrm{P}<0.05$ was considered to 
Table II. Responses of CRT and FP.

\begin{tabular}{lccccc}
\hline Responses & CR & PR & SD & PD & P-value \\
\hline CRT & & & & & \\
NLR $>3$ & 0 & $11(19.3 \%)$ & $18(31.6 \%)$ & $28(49.1)^{\mathrm{a}}$ & 57 \\
NLR $\leq 3$ & $1(1.9 \%)$ & $29(54.7 \%)^{\mathrm{a}}$ & $15(28.3 \%)$ & $8(15.1 \%)$ & 53 \\
FP & 0 & $8(13.1 \%)$ & $24(39.3 \%)$ & $29(47.6 \%)^{\mathrm{a}}$ & 61 \\
NLR $>3$ & 0 & $35(39.3 \%)^{\mathrm{a}}$ & $37(41.6 \%)$ & $17(19.1 \%)$ & 89 \\
NLR $\leq 3$ & & & & $<0.05$ \\
\hline
\end{tabular}

Judged by RECIST criteria. CRT, chemoradiation therapy; FP, 5-fluorouracil and cisplatin; CR, complete remission; PR, partial remission; SD, stable disease; $\mathrm{PD}$, progressive disease; NLR, neutrophil-to-lymphocyte ratio. ${ }^{\mathrm{a}} \mathrm{P}<0.05$.
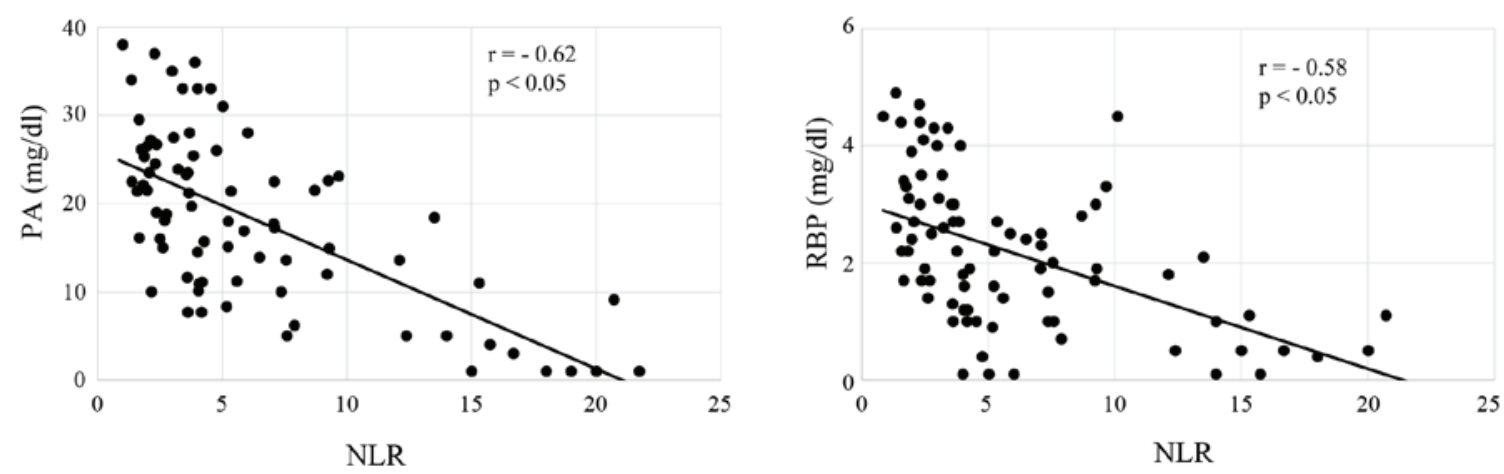

Figure 1. Correlation of NLR with nutritional parameters in patients receiving chemoradiation therapy. NLR, neutrophil to lymphocyte ratio; PA, prealbumin; RBP, retinol binding protein.
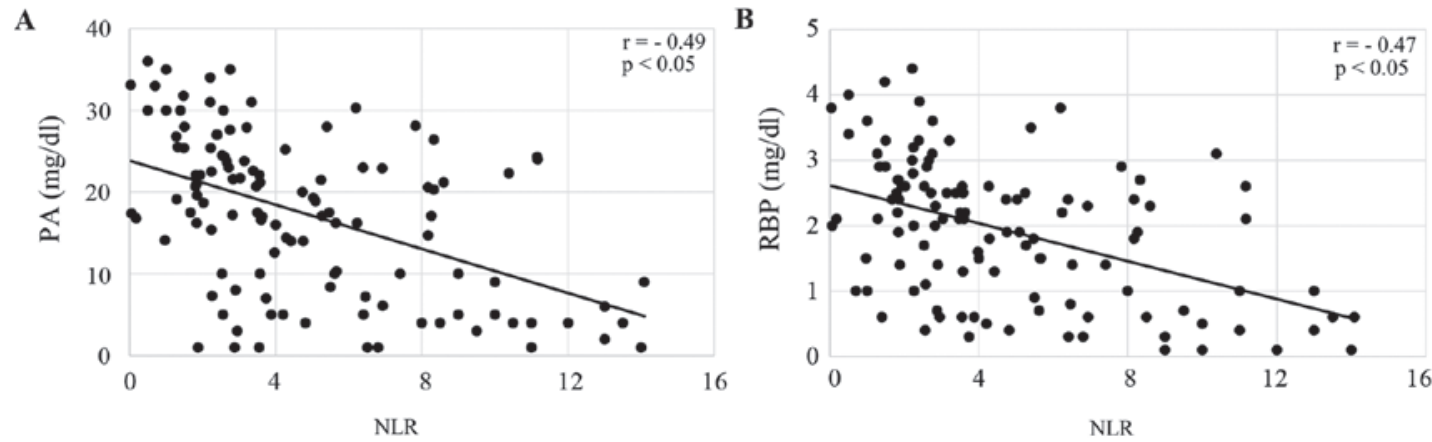

Figure 2. Correlation of NLR with (A) prealbumin and (B) retinol binding protein in patients receiving a combination of 5-fluorouracil and cisplatin. NLR, neutrophil to lymphocyte ratio; PA, prealbumin; RBP, retinol binding protein.

indicate a statistically significant difference. The patients were divided into two groups according to their NLR: High NLR (NLR >3.0) and low NLR (NLR $\leq 3.0)$. Survival and response to chemotherapy were compared between groups. OS was defined as the time from the start of chemotherapy until death.

\section{Results}

All of the enrolled patients were divided according to their NLR, and the correlation of NLR with nutritional markers, including PA, RBP, CRP and SI, was analyzed. Serum levels of PA and RBP were both significantly inversely correlated with NLR in patients treated with CRT (both $\mathrm{P}<0.05$; Fig. $1 \mathrm{~A}$ and $\mathrm{B}$ ), and patients treated with $\mathrm{FP}$ (both $\mathrm{P}<0.05$; Fig. $2 \mathrm{~A}$ and $\mathrm{B}$ ). CRP levels were significantly correlated, and SI was inversely correlated with NLR in CRT patients (both $\mathrm{P}<0.05$; Fig. $3 \mathrm{~A}$ and $\mathrm{B}$ ) and FP patients (both $\mathrm{P}<0.05$; Fig. $4 \mathrm{~A}$ and $\mathrm{B}$ ). The responses to CRT and FP according to RECIST criteria are presented in Table II. In patients treated with CRT, a partial response was significantly higher in patients with a low NLR (19.3\% vs. 54.7\%; P<0.05; Table II) and with progressive disease compared to those with a high NLR (49.1\% vs. $15.1 \%$; $\mathrm{P}<0.05$; Table II). In patients treated with FP, a partial response was also significantly higher in patients with a low NLR (13.1\% vs. $39.7 \%$; $\mathrm{P}<0.05$; Table II) and with progressive disease compared to those with a high NLR (47.6\% vs. 19.1\%; P<0.05; Table II). Fig. 5 demonstrates 

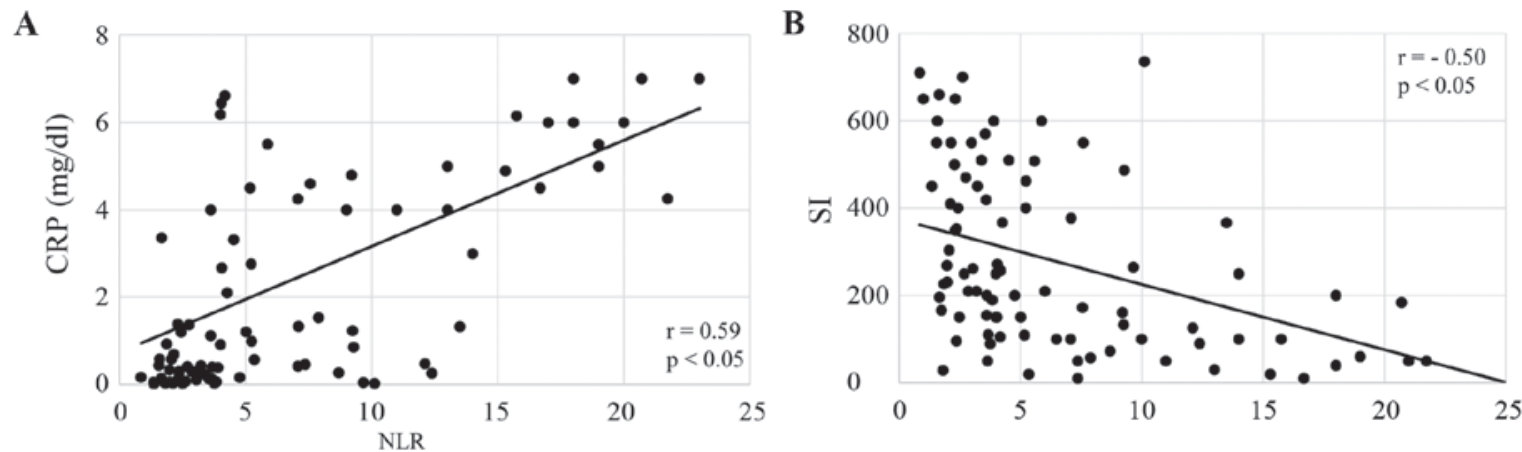

Figure 3. Correlation of NLR with (A) inflammation and (B) cell-mediated immune function in patients received chemoradiation therapy. CRP, C reactive protein; SI, stimulation index; NLR, neutrophil to lymphocyte ratio.
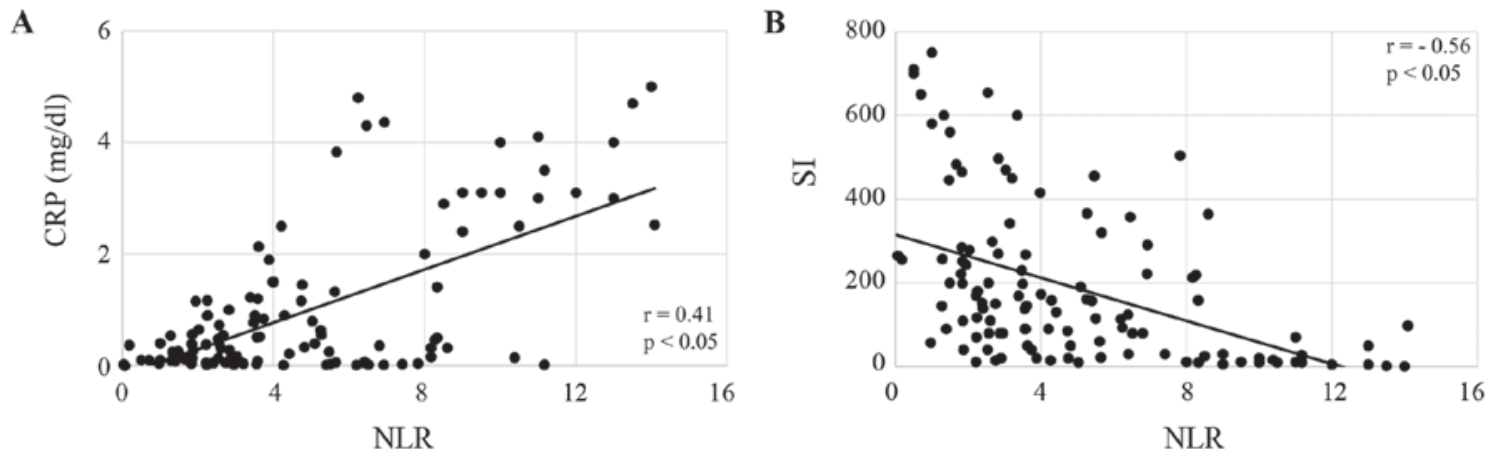

Figure 4. Correlation of NLR with (A) inflammation and (B) cell-mediated immune function in patients received a combination of 5-fluorouracil and cisplatin. NLR, neutrophil to lymphocyte ratio; CRP, C reactive protein; SI, stimulation index.
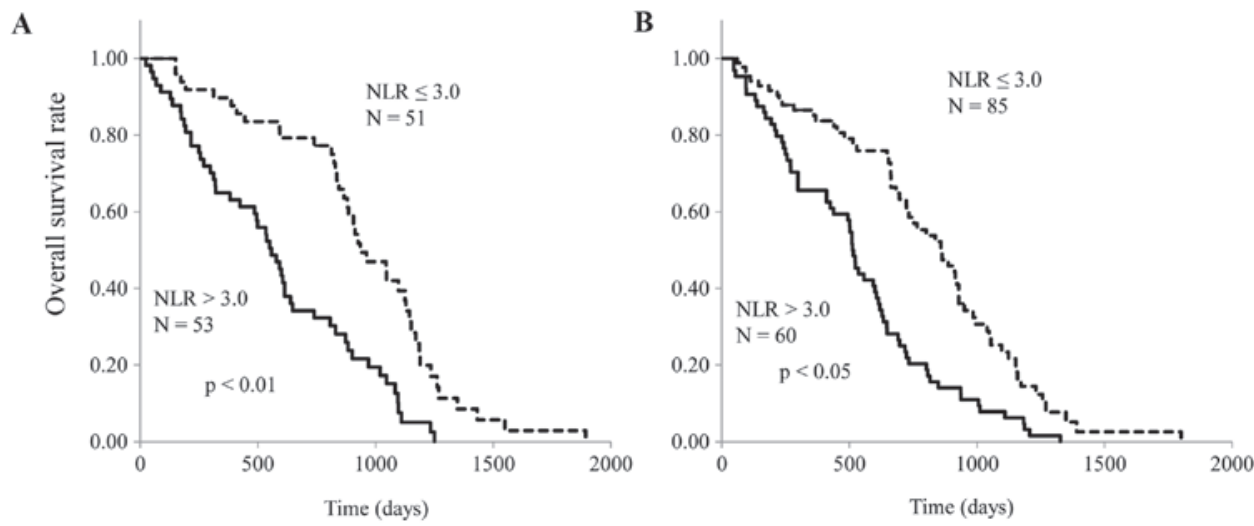

Figure 5. Kaplan-Meier curves for patients with low and high neutrophil to lymphocyte ratio. NLR, neutrophil to lymphocyte ratio.

the relationship between NLR and OS in patients with ESCC treated with CRT and FP. OS of patients with CRT and FP were both significantly worse in patients with a high NLR than in those with a low NLR (Fig. 5; $\mathrm{P}<0.01$ and $\mathrm{P}<0.05$, respectively).

\section{Discussion}

In the present study, the ability of NLR to serve as a prognostic indicator of survival in patients with locally advanced and metastatic ESCC treated with CRT or FP was analyzed. The results demonstrated that NLR was a useful marker of malnutrition, systemic inflammation and immune suppression, and was also a strong prognostic indicator.
Various inflammation-related markers such as CRP, NLR and GPS are influenced by the production of pro- and anti-inflammatory cytokines, and are associated with a poor prognosis in various types of cancer (22-24). Immune suppression was closely associated with increased NLR as the levels of SI were significantly inversely correlated to increased NLR. The authors previously reported that increasing levels of circulating myeloid-derived suppressor cells (MDSCs) correlate with decreased SI, malnutrition, and increased vascular endothelial growth factor (VEGF) levels in patients with cancer $(36,37)$. The authors speculated that immune suppression may also occur with increased MDSC levels, activated by inflammation or tumor-produced VEGF. 
It remains unclear why tumor responses to chemotherapy are associated with NLR or systemic inflammation. Because malnutrition, immune suppression and PS are closely associated with one other, patients with a fair PS may be able to receive higher doses of chemotherapeutic drugs or a dose-dense chemotherapy schedule. Another possibility was recently raised. Immunogenic cell death after chemotherapy or radiotherapy has been reported $(38,40,41)$. Moreover, it is possible that lymphocytes in cancer-bearing hosts can recognize neoantigens, tumor-specific mutation-derived antigens (42). Because immune function depends upon systemic inflammation, the anti-tumor activity of cytotoxic T lymphocytes that recognize tumor cells may be suppressed in patients with high inflammation, and the response to chemotherapy and the prognosis of these patients may be poor.

In conclusion, the results of the present study suggested that NLR may serve as a useful marker of the tumor response, immune suppression, malnutrition and prognosis upon CRT or FP in patients with locally advanced or metastatic ESCC. However, because NLR is easily influenced by infection or stress, a useful cut-off level needs to be investigated in clinical practice. Furthermore, a combination of anti-inflammatory with chemotherapeutic agents may have therapeutic benefits in ESCC; thus, further studies in this area are needed.

\section{References}

1. Ferlay J, Soerjomataram I, Dikshit R, Eser S, Mathers C, Rebelo M, Parkin DM, Forman D and Bray F: Cancer incidence and mortality worldwide: Sources, methods and major patterns in GLOBOCAN 2012. Int J Cancer 136: E359-E386, 2015.

2. Sohda M and Kuwano H: Current status and future prospects for esophageal cancer treatment. Ann Thorac Cardiovasc Surg 23: 1-11, 2017.

3. Coussens LM and Werb Z: Inflammation and cancer. Nature 420: 860-867, 2002

4. Balkwill $\mathrm{F}$ and Mantovani A: Inflammation and cancer: Back to Virchow? Lancet 357: 539-545, 2001.

5. Jaiswal M, LaRusso NF, Burgart LJ and Gores GJ: Inflammatory cytokines induce DNA damage and inhibit DNA repair in cholangiocarcinoma cells by a nitric oxide-dependent mechanism. Cancer Res 60: 184-190, 2000.

6. McMillan DC: The systemic inflammation-based Glasgow Prognostic Score: A decade of experience in patients with cancer. Cancer Treat Rev 39: 534-540, 2013

7. Smith RA, Bosonnet L, Raraty M, Sutton R, Neoptolemos JP, Campbell F and Ghaneh P: Preoperative platelet-lymphocyte ratio is an independent significant prognostic marker in resected pancreatic ductal adenocarcinoma. Am J Surg 197: 466-472, 2009.

8. Tomita M, Shimizu T, Ayabe T, Yonei A and Onitsuka T: Preoperative neutrophil to lymphocyte ratio as a prognostic predictor after curative resection for non-small cell lung cancer. Anticancer Res 31: 2995-2998, 2011.

9. Pinato DJ, Shiner RJ, Seckl MJ, Stebbing J, Sharma R and Mauri FA: Prognostic performance of inflammation-based prognostic indices in primary operable non-small cell lung cancer. $\mathrm{Br}$ J Cancer 110: 1930-1935, 2014.

10. Zhang H, Zhang L, Zhu K, Shi B, Yin Y, Zhu J, Yue D, Zhang B and Wang C: Prognostic significance of combination of preoperative platelet count and neutrophil-lymphocyte ratio (COP-NLR) in patients with non-small cell lung cancer: Based on a large cohort study. PLoS One 10: e0126496, 2015.

11. Shibutani M, Maeda K, Nagahara H, Ohtani H, Iseki Y, Ikeya T, Sugano K and Hirakawa K: The prognostic significance of a postoperative systemic inflammatory response in patients with colorectal cancer. World J Surg Oncol 13: 194, 2015.

12. Albayrak S, Zengin K, Tanik S, Atar M, Unal SH, Imamoglu MA and Gurdal M: Can the neutrophil-to-lymphocyte ratio be used to predict recurrence and progression of non-muscle-invasive bladder cancer? Kaohsiung J Med Sci 32: 327-333, 2016.
13. Dong YW, Shi YQ, He LW and Su PZ: Prognostic significance of neutrophil-to-lymphocyte ratio in rectal cancer: A meta-analysis. Onco Targets Ther 9: 3127-3134, 2016.

14. Nakamura K, Nagasaka T, Nishida T, Haruma T, Ogawa C, Kusumoto T, Seki N and Hiramatsu Y: Neutrophil to lymphocyte ratio in the pre-treatment phase of final-line chemotherapy predicts the outcome of patients with recurrent ovarian cancer. Oncol Lett 11: 3975-3981, 2016.

15. Aino H, Sumie S, Niizeki T, Kuromatsu R, Tajiri N, Nakano M, Satani M, Okamura S, Shimose S, Miyahara K, et al: The systemic inflammatory response as a prognostic factor for advanced hepatocellular carcinoma with extrahepatic metastasis. Mol Clin Oncol 5: 83-88, 2016.

16. Ding PR, An X, Zhang RX, Fang YJ, Li LR, Chen G, Wu XJ, Lu ZH, Lin JZ, Kong LH, et al: Elevated preoperative neutrophil to lymphocyte ratio predicts risk of recurrence following curative resection for stage IIA colon cancer. Int J Colorectal Dis 25: 1427-1433, 2010.

17. Jung MR, Park YK, Jeong O, Seon JW, Ryu SY, Kim DY and Kim YJ: Elevated preoperative neutrophil to lymphocyte ratio predicts poor survival following resection in late stage gastric cancer. J Surg Oncol 104: 504-510, 2011.

18. Stotz M, Gerger A, Eisner F, Szkandera J, Loibner H, Ress AL, Kornprat P, AlZoughbi W, Seggewies FS, Lackner C, et al: Increased neutrophil-lymphocyte ratio is a poor prognostic factor in patients with primary operable and inoperable pancreatic cancer. Br J Cancer 109: 416-421, 2013.

19. Li Y, An J, Huang S, He J and Zhang J: Esophageal cancer-derived microvesicles induce regulatory B cells. Cell Biochem Funct 33: 308-313, 2015.

20. Gholamin M, Moaven O, Memar B, Farshchian M, Naseh H, Malekzadeh R, Sotoudeh M, Rajabi-Mashhadi MT, Forghani MN, Farrokhi F, et al: Overexpression and interactions of interleukin-10, transforming growth factor beta, and vascular endothelial growth factor in esophageal squamous cell carcinoma. World J Surg 33: 1439-1445, 2009.

21. Chen MF, Chen PT, Chen WC, Lu MS, Lin PY and Lee KD: The role of PD-L1 in the radiation response and prognosis for esophageal squamous cell carcinoma related to IL-6 and T-cell immunosuppression. Oncotarget 7: 7913-7924, 2016.

22. Grivennikov SI, Greten FR and Karin M: Immunity, inflammation, and cancer. Cell 140: 883-899, 2010.

23. McMillan DC: Systemic inflammation, nutritional status and survival in patients with cancer. Curr Opin Clin Nutr Metab Care 12: 223-226, 2009.

24. Zahorec R: Ratio of neutrophil to lymphocyte counts - rapid and simple parameter of systemic inflammation and stress in critically ill. Bratisl Lek Listy 102: 5-14, 2001.

25. Higuchi K, Koizumi W, Tanabe S, Sasaki T, Katada C, Azuma M, Nakatani K, Ishido K, Naruke A and Ryu T: Current management of esophageal squamous-cell carcinoma in Japan and other countries. Gastrointest Cancer Res 3: 153-161, 2009.

26. Herskovic A, Martz K, al-Sarraf M, Leichman L, Brindle J, Vaitkevicius V, Cooper J, Byhardt R, Davis L and Emami B: Combined chemotherapy and radiotherapy compared with radiotherapy alone in patients with cancer of the esophagus. N Engl J Med 326: 1593-1598, 1992.

27. Ishida K, Iizuka T, Ando N and Ide H: Phase II study of chemoradiotherapy for advanced squamous cell carcinoma of the thoracic esophagus: Nine Japanese institutions trial. Jpn J Clin Oncol 26: 310-315, 1996.

28. Finn OJ: Cancer immunology. N Engl J Med 358: 2704-2715, 2008.

29. Yoshimoto Y, Suzuki Y, Mimura K, Ando K, Oike T, Sato H, Okonogi N, Maruyama T, Izawa S, Noda SE, et al: Radiotherapy-induced anti-tumor immunity contributes to the therapeutic efficacy of irradiation and can be augmented by CTLA-4 blockade in a mouse model. PLoS One 9: e92572, 2014.

30. Lee Y, Auh SL, Wang Y, Burnette B, Wang Y, Meng Y, Beckett M, Sharma R, Chin R, Tu T, et al: Therapeutic effects of ablative radiation on local tumor require $\mathrm{CD} 8^{+} \mathrm{T}$ cells: Changing strategies for cancer treatment. Blood 114: 589-595, 2009.

31. Takeshima T, Chamoto K, Wakita D, Ohkuri T, Togashi Y, Shirato H, Kitamura $\mathrm{H}$ and Nishimura T: Local radiation therapy inhibits tumor growth through the generation of tumor-specific CTL: Its potentiation by combination with Th1 cell therapy. Cancer Res 70: 2697-2706, 2010.

32. Shibata M and Gonda K: Cachexia, immunological participation. In: Horizons in Cancer Research. Nova Science. Watanabe HS (ed). Publishers Inc., New York, pp19-32, 2015. 
33. Shibata M, Nezu T, Kanou H, Abe H, Takekawa M and Fukuzawa M: Decreased production of interleukin-12 and type 2 immune responses are marked in cachectic patients with colorectal and gastric cancer. J Clin Gastroenterol 34: 416-420, 2002.

34. Shibata M, Nagata Y, Kimura T, Kanou H, Nezu T, Takekawa M and Fukuzawa M: Elevated serum concentration of interleukin-1 receptor antagonist (IL-1ra) is correlated to interleukin-6 and hypoalbuminemia in cachectic patients with colorectal cancer. Int J Clin Oncol 5: 116-120, 2000.

35. Shibata $M$ and Takekawa $M$ : Increased serum concentration of circulating soluble receptor for interleukin-2 and its effect as a prognostic indicator in cachectic patients with gastric and colorectal cancer. Oncology 56: 54-58, 1999.

36. Ohki S, Shibata M, Gonda K, Machida T, Shimura T, Nakamura I, Ohtake T, Koyama Y, Suzuki S, Ohto H, et al: Circulating myeloid-derived suppressor cells are increased and correlated to immune suppression and hypoalbuminemia in patients with cancer. Oncol Rep 28: 453-458, 2012.

37. Nakamura I, Shibata M, Gonda K, Yazawa T, Shimura T, Anazawa T, Suzuki S, Sakurai K, Koyama Y, Ohto H, et al: Serum levels of vascular endothelial growth factor are increased and correlate with malnutrition, immunosuppression involving MDSCs and systemic inflammation in patients with cancer of the digestive system. Oncol Lett 5: 1682-1686, 2013.
38. Suzuki Y, Mimura K, Yoshimoto Y, Watanabe M, Ohkubo Y, Izawa S, Murata K, Fujii H, Nakano T and Kono K: Immunogenic tumor cell death induced by chemoradiotherapy in patients with esophageal squamous cell carcinoma. Cancer Res 72: 3967-3976, 2012.

39. Eisenhauer EA, Therasse P, Bogaerts J, Schwartz LH, Sargent D, Ford R, Dancey J, Arbuck S, Gwyther S, Mooney M, et al: New response evaluation criteria in solid tumours: Revised RECIST guideline (version 1.1). Eur J Cancer 45: 228-247, 2009.

40. Yoshimoto Y, Kono K and Suzuki Y: Anti-tumor immune responses induced by radiotherapy. Fukushima J Med Sci 61: 13-22, 2015.

41. Garg AD, Galluzzi L, Apetoh L, Baert T, Birge RB, Bravo-San Pedro JM, Breckpot K, Brough D, Chaurio R, Cirone M, et al: Molecular and translational classifications of DAMPs in immunologic cell death. Front Immunol 6: 588, 2015.

42. Wang RF and Wang HY: Immune targets and neoantigens for cancer immunotherapy and precision medicine. Cell Res 27: 11-37, 2017 